\title{
Abnormal Exacerbation of Moderately Differentiated Gastric Adenocarcinoma in a Patient with TAFRO Syndrome: An Impaired Tumor Immunity?
}

\author{
Tadaaki Inano $^{a}$ Hajime Yasuda $^{a}$ Yutaka Tsukune $^{a}$ Miyuki Tsutsui ${ }^{a}$ \\ Nadila Wali $^{b}$ Harumi Saeki $^{\text {, c }}$ Kazunori Kajino ${ }^{\text {, c }}$ Okio Hino $^{\text {b }}$ \\ Yasufumi Masakid $^{d}$ Norio Komatsua, e, f \\ aDepartment of Hematology, Juntendo University School of Medicine, Tokyo, Japan; \\ ${ }^{b}$ Department of Pathology and Oncology, Juntendo University School of Medicine, Tokyo, \\ Japan; 'Department of Human Pathology, Juntendo University School of Medicine, Tokyo,

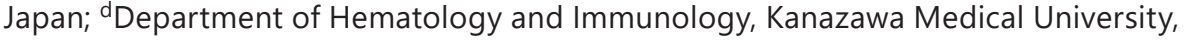 \\ Uchinada, Japan; 'Laboratory for the Development of Therapies Against MPN, Juntendo \\ University Graduate School of Medicine, Tokyo, Japan; ' Department of Advanced \\ Hematology, Juntendo University Graduate School of Medicine, Tokyo, Japan
}

Keywords

Anti-PD-1/PD-L1 - Gastric cancer · Hyperprogressive disease $\cdot T$ follicular helper cells · Tumor immunity

\begin{abstract}
TAFRO syndrome is a relatively new disease entity first reported in 2010. We report a case of TAFRO syndrome accommodated by abnormal exacerbation of moderately differentiated gastric adenocarcinoma. The pathophysiology of TAFRO syndrome is largely unknown, but because the disease often responds to immunosuppressive therapy and also because $T$ follicular helper (Tfh) cells are reported to be drastically decreased in TAFRO syndrome, involvement of a dysregulated immune system can be speculated. Growing evidence points toward a pivotal role of Th cells in tumor immunity through supporting ectopic lymphoid structures, which are recruitment sites for cells directly engaging in antitumor activity such as $\mathrm{CD} 8^{+} \mathrm{T}$ cells, NK cells, and macrophages. In fact, Tfh cells are reported to positively correlate with longer survival in human colorectal and breast cancer. Combined with our observations of hyperprogressive gastric cancer in the presented patient, an impaired tumor immunity is strongly indicated in TAFRO syndrome.
\end{abstract}




\section{Introduction}

TAFRO syndrome is a systemic inflammatory disorder first reported by Takai et al. in 2010[1]. Major manifestations are thrombocytopenia, anasarca, fever, renal insufficiency, organomegaly, and myelofibrosis [2,3]. The pathophysiology of TAFRO syndrome is unknown. Lymph node histopathology of TAFRO syndrome resembles that of Castleman disease, and thus some researchers consider TAFRO syndrome as a variant of idiopathic multicentric Castleman disease, whereas others consider TAFRO syndrome as a distinct entity because of differing clinical features. Specific markers unique to TAFRO syndrome are currently lacking, and excluding other disorders that mimic TAFRO syndrome such as autoimmune disease, infections, and lymphoma is an important part of the diagnostic process. The median age of patients with TAFRO syndrome is reported to be around 50-59 years, and there is no male/ female predominance. The clinical course is acute to subacute, and the 5-year survival rate is reported to be dismal at $66.5 \%[4,5]$. We report a TAFRO patient accommodated by abnormally accelerated exacerbation of moderately differentiated gastric adenocarcinoma. $\mathrm{T}$ follicular helper (Tfh) cells are reported to be drastically decreased in TAFRO syndrome, and Tfh cells are known to play a major role in tumor immunity through supporting ectopic lymphoid structures, which are recruitment sites for cells directly engaging in antitumor activity such as $\mathrm{CD}^{+} \mathrm{T}$ cells, NK cells, and macrophages. Thus, reduced Tfh cells can lead to impaired tumor immunity, and our observations strongly support the abovementioned findings and shed further light to the pathophysiology of TAFRO syndrome.

\section{Case Presentation}

An 86-year-old man was referred to Juntendo University Hospital due to fever, appetite loss, edema, and pancytopenia. He had a history of chronic obstructive pulmonary disease, type-2 diabetes mellitus, and right-lobe hepatectomy for which the reason was unknown. Computed tomography (CT) revealed bilateral pleural effusion, multiple mediastinal lymph node swellings which were around $10-20 \mathrm{~mm}$ in diameter, and splenomegaly. He presented with a white blood cell count of $2.1 \times 10^{9} / \mathrm{L}$, hemoglobin of $6.9 \mathrm{~g} / \mathrm{dL}$, and platelet count of 14 $\times 10^{9} / \mathrm{L}$. Serum creatinine and CRP were elevated at $1.33 \mathrm{mg} / \mathrm{dL}$ and $3.9 \mathrm{mg} / \mathrm{dL}$, and LDH and ALP levels were normal. Serum IgG, IgG4, and IL-6 levels were increased at 2,496 mg/dL, 426 $\mathrm{mg} / \mathrm{dL}$, and $459 \mathrm{pg} / \mathrm{mL}$, respectively. Peripheral blood screening tests were negative for human immunodeficiency virus and human herpesvirus-8, and Epstein-Barr viral status indicated a past infection with undetectable VCA-IgM, VCA-IgG of 1:160, and EBNA of 1:160. Rheumatoid factor was slightly elevated at $23.3 \mathrm{IU} / \mathrm{mL}$, but elevations were absent concerning anti-CCP antibodies, anti-nuclear antibodies, anti-ds-DNA antibodies, MPO-ANCA, and PR3-ANCA. Anti-SS-A antibodies, anti-SS-B antibodies, and interferon-gamma release assays $\left(\mathrm{T}_{-S P O T}{ }^{\circledR}\right.$ ) were also negative. Immunoelectrophoresis revealed no M-protein. Bone marrow (BM) aspiration was poor and BM biopsy showed myeloid hyperplasia and myelofibrosis (MF-2) (Fig. 1a, b). BM pathology did not meet the criteria of primary myelofibrosis, and $J A K 2 \mathrm{~V} 617 \mathrm{~F}, C A L R$, and MPL mutations were found to be absent by methods previously reported [6]. Transbronchial lung biopsy of mediastinal lymph nodes was carried out, but the acquired tissue was insufficient material. The patient was diagnosed with TAFRO syndrome, according to the criteria proposed by Masaki et al. [2, 3]. Treatment with prednisolone $20 \mathrm{mg} / \mathrm{day}$ $(0.5 \mathrm{mg} / \mathrm{kg} / \mathrm{day})$ was initiated on day 50 of admission, continued for 7 weeks, and slowly tapered thereafter. Prednisolone therapy was effective, with platelet counts exceeding $100 \times 10^{9} / \mathrm{L}$ and his general condition recovered remarkably. Day 93 CT scans revealed abnormal gastric wall thickening and multiple hepatic tumors which were not detected upon admission (Fig. 2),

\section{Karger'}




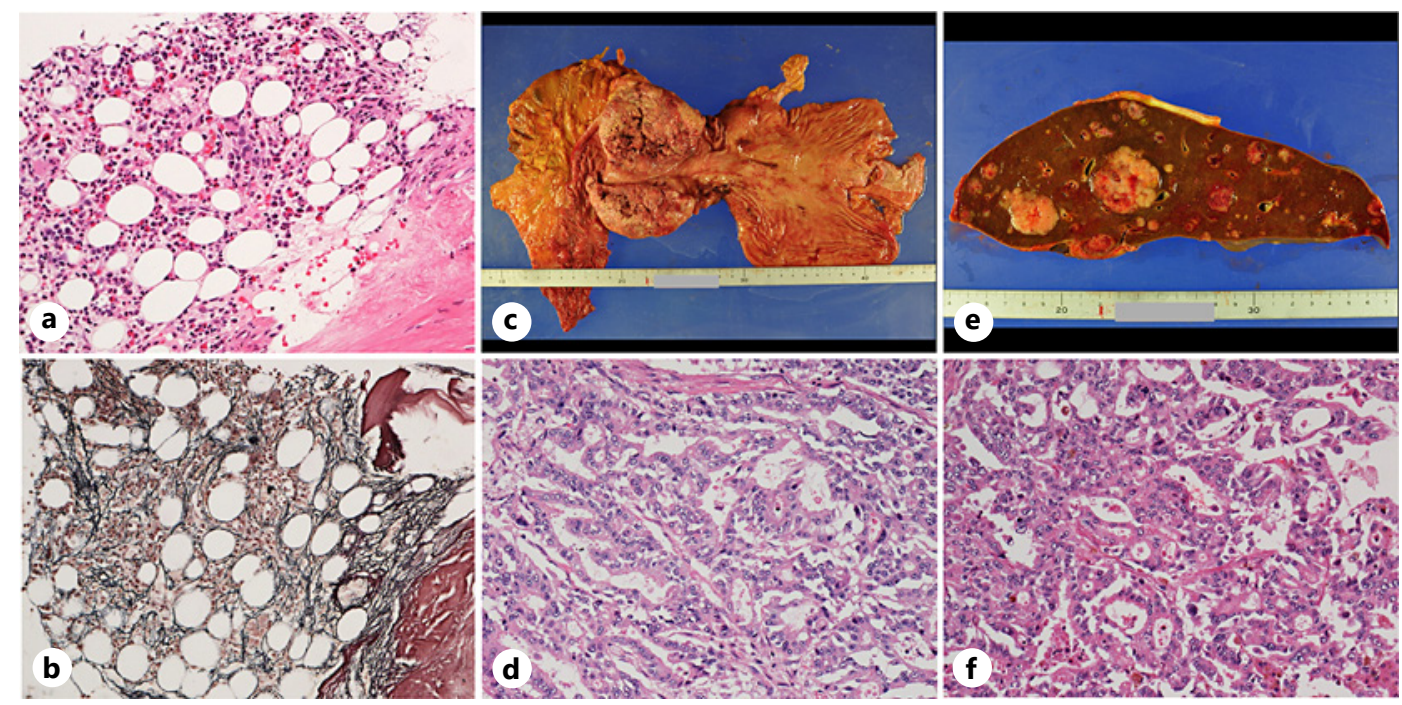

Fig. 1. Bone marrow hematoxylin and eosin staining (a) and silver staining (b). c-f Autopsy results showing macroscopic and microscopic pathology. $\mathbf{c}, \mathbf{d}$ Moderately differentiated adenocarcinoma in the gastric antrum. $\mathbf{e}, \mathbf{f}$ Metastatic lesions of moderately differentiated adenocarcinoma in the liver.

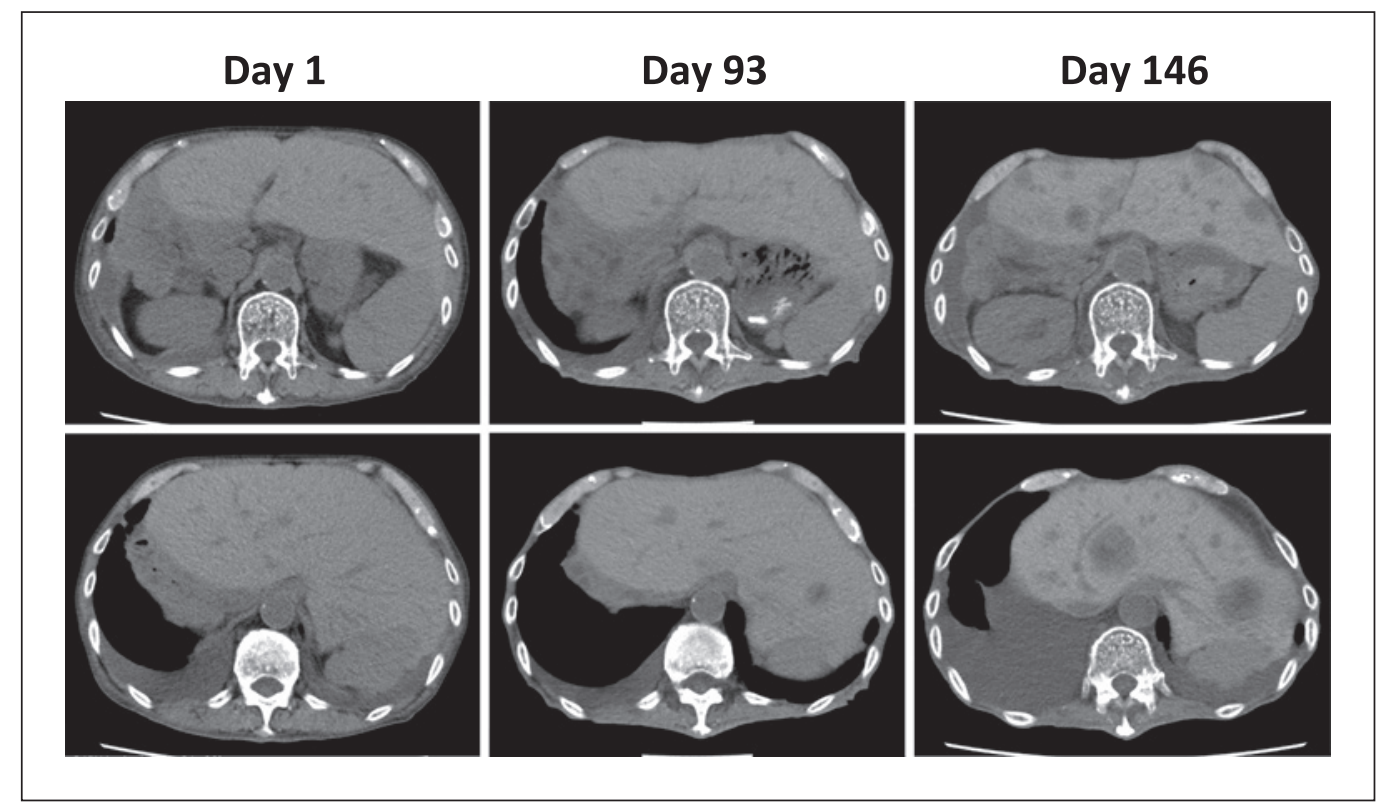

Fig. 2. Transition of hepatic metastasis of gastric adenocarcinoma upon CT scans. Days are shown as days after admission.

and his general condition began to deteriorate again around day 102. Although he was still on $18 \mathrm{mg} /$ day of prednisolone therapy, high fever recurred and platelet counts decreased to around $10 \times 10^{9} / \mathrm{L}$. Follow-up CT scans on day 146 showed prominent progression of the gastric and hepatic tumors (Fig. 2), and he expired on day 159. Autopsy revealed moderately differentiated gastric adenocarcinoma in the antrum measuring $80 \times 70 \times 55 \mathrm{~mm}$ with extensive metastasis involving the liver, lungs, and adrenal glands (Fig. 1c-f), which was thought to be the direct cause of death. Immunohistochemistry of the gastric cancer showed 
negativity for PD-L1. Lymphadenopathy seen at disease onset was absent upon autopsy, probably due to the long-term steroid therapy. Peritoneal and pleural dissemination of adenocarcinoma were not observed, and thus the pleural effusion was confirmed not to be due to carcinomatosis.

\section{Discussion/Conclusion}

The gastric cancer and hepatic metastasis were first detected by day 93 CT scans, and follow-up CT scans on day 146 showed prominent exacerbation of the lesions, which is clearly abnormal disease progression for moderately differentiated adenocarcinoma. This is the first report of TAFRO syndrome accommodated by a concomitant solid tumor, and we suspect that TAFRO syndrome contributed to the abnormal exacerbation of gastric cancer. Abnormal exacerbations of cancers are known as "hyperprogressive disease" and are reported to occur in a subset of cancer patients treated with immunotherapy including PD-1/PD-L1 inhibitors and IFN- $\alpha$, implicating that an altered immune status contributes to the etiology of this phenomenon [7]. The pathophysiology of TAFRO syndrome is unknown [8], but because the disease often responds to immunosuppressive therapy and also because Tfh cells are reported to be drastically decreased in TAFRO syndrome, involvement of a dysregulated immune system can be speculated [9]. Growing evidence points toward a pivotal role of Tfh cells in tumor immunity through supporting ectopic lymphoid structures, which are recruitment sites for cells directly engaging in antitumor activity such as $\mathrm{CD}^{+} \mathrm{T}$ cells, NK cells, and macrophages [10]. In fact, Tfh cells have been reported to positively correlate with longer survival in human colorectal and breast cancer [10]. Combined with our observations of hyperprogressive disease in the presented patient, an impaired tumor immunity is strongly indicated in TAFRO syndrome.

In 2016, Masaki et al. [2] and Iwaki et al. [11] published different diagnostic criteria for TAFRO syndrome, and the latter necessitates characteristic pathological findings on lymph node biopsy [3]. However, as Masaki et al. [2] have pointed out, lymphadenopathy is modest in TAFRO syndrome, and targeting such small lymph nodes for pathological assessment is often difficult, as was the case in our patient. TAFRO syndrome largely relies on a diagnosis of exclusion. Our case not only fulfilled the diagnostic criteria for TAFRO published by Masaki et al. [2], but antemortem screening tests and autopsy confirmed no other causes of the clinical manifestations.

In conclusion, impaired tumor immunity is strongly suspected in TAFRO syndrome, but whether this dysregulation is the cause or result of TAFRO syndrome is unknown. Further accumulation of cases is necessary to validate our findings.

\section{Acknowledgments}

We thank the members of the Department of Hematology, Department of Pathology and Oncology, and Department of Human Pathology, Juntendo University School of Medicine, for encouraging this study.

\section{Statement of Ethics}

This study was approved by the Ethics Committee of Juntendo University School of Medicine (IRB\#2015122). Written informed consent was obtained from the patient for publication of the details of the medical case and accompanying images.

\section{Karger'}




\section{Conflict of Interest Statement}

The authors declare that they have no conflicts of interest.

\section{Funding Sources}

The authors received no financial support for the authorship.

\section{Author Contributions}

T.I. and H.Y. wrote the manuscript. Y.T., M.T., N.W., H.S., K.K., and O.H. collected and interpreted the data. N.K. and Y.M. revised the manuscript and gave final approval.

\section{Data Availability Statement}

Data are available on request.

\section{References}

1 Takai K, Nikkuni K, Shibuya H, Hashidate H. Thrombocytopenia with mild bone marrow fibrosis accompanied by fever, pleural effusion, ascites and hepatosplenomegaly. Rinsho Ketsueki. 2010;51(5):320-5.

2 Masaki Y, Kawabata H, Takai K, Kojima M, Tsukamoto N, Ishigaki Y, et al. Proposed diagnostic criteria, disease severity classification and treatment strategy for TAFRO syndrome, 2015 version. Int J Hematol. 2016;103(6): 686-92.

3 Masaki Y, Kawabata H, Takai K, Tsukamoto N, Fujimoto S, Ishigaki Y, et al. 2019 updated diagnostic criteria and disease severity classification for TAFRO syndrome. Int J Hematol. 2020;111(1):155-8.

4 Fujimoto S, Sakai T, Kawabata H, Kurose N, Yamada S, Takai K, et al. Is TAFRO syndrome a subtype of idiopathic multicentric Castleman disease? Am J Hematol. 2019;94(9):975-83.

5 Igawa T, Sato Y. TAFRO syndrome. Hematol Oncol Clin North Am. 2018;32(1):107-18.

6 Misawa K, Yasuda H, Araki M, Ochiai T, Morishita S, Shirane S, et al. Mutational subtypes of JAK2 and CALR correlate with different clinical features in Japanese patients with myeloproliferative neoplasms. Int J Hematol. 2018;107(6):673-80.

7 Han XJ, Alu A, Xiao YN, Wei YQ, Wei XW. Hyperprogression: a novel response pattern under immunotherapy. Clin Transl Med. 2020;10(5):e167.

8 Kageyama C, Igawa T, Gion Y, Iwaki N, Tabata T, Tanaka T, et al. Hepatic Campylobacter jejuni infection in patients with Castleman-Kojima disease (idiopathic multicentric Castleman disease with thrombocytopenia, anasarca, fever, reticulin fibrosis, and organomegaly (TAFRO) syndrome). Pathol Int. 2019;69(10):572-9.

9 Kurose N, Guo X, Shioya A, Mizutani KI, Kumagai M, Fujimoto S, et al. The potential role of follicular helper T cells in idiopathic multicentric Castleman disease with and without TAFRO syndrome. Pathol Res Pract. 2019; 215(10):152563.

10 Crotty ST. Follicular helper cell biology: a decade of discovery and diseases. Immunity. 2019;50(5):1132-48.

11 Iwaki N, Fajgenbaum DC, Nabel CS, Gion Y, Kondo E, Kawano M, et al. Clinicopathologic analysis of TAFRO syndrome demonstrates a distinct subtype of HHV-8-negative multicentric Castleman disease. Am J Hematol. 2016;91(2):220-6. 